\title{
Solutions of D-dimensional Schrodinger equation for Woods- Saxon potential with spin-orbit, coulomb and centrifugal terms through a new hybrid numerical fitting Nikiforov-Uvarov method
}

\author{
A. Niknam ${ }^{1}$ - A. A. Rajabi ${ }^{1}$ M. Solaimani ${ }^{2}$
}

Received: 26 September 2015/Accepted: 9 November 2015/Published online: 9 December 2015

(C) The Author(s) 2015. This article is published with open access at Springerlink.com

\begin{abstract}
Solution of the radial Schrodinger equation for the Woods-Saxon potential together with spin-orbit interaction, coulomb and centrifugal terms by using usual Nikiforov-Uvarov (NU) method is not possible. Here, we have presented a new NU procedure with which we are able to solve this Schrodinger equation and any other onedimensional ones with any shape of the potential profile. For this purpose, we have combined the NU method with numerical fitting schema. The energy eigenvalues and corresponding eigenfunctions for various values of $n, 1$, and $\mathrm{j}$ quantum numbers have been obtained. Good agreement with experimental values is also achieved. We have calculated the $1 / 2^{+}$state energy with more accuracy (our absolute error $=0.023 \mathrm{MeV}$ and Hagen et al. absolute error $=0.0918 \mathrm{MeV}$ ), while Hagen et al. have calculated the $5 / 2^{+}$state energy with higher accuracy (our absolute error $=0.71 \mathrm{MeV}$ and Hagen et al. absolute error $=$ $0.0337 \mathrm{MeV}$ ). Our wave functions are in agreement with Kim et al.'s work, too.
\end{abstract}

Keywords Nikiforov-Uvarov (NU) method .

Eigenvalues and Eigenfunctions - Woods-Saxon potential . Spin-orbit interaction $\cdot$ Coulomb potential $\cdot$ Centrifugal term $\cdot$ Numerical fitting

A. Niknam

niknamabbas@gmail.com

1 Physics Faculty, Shahrood University, P.O. Box 3619995161-316, Shahrood, Iran

2 Department of Physics, Faculty of Science, Qom University of Technology, Qom, Iran

\section{Introduction}

In the study of the breakup of ${ }^{17} \mathrm{~F}$ into proton $+{ }^{16} \mathrm{O}$, some potential model for ${ }^{17} \mathrm{~F}$ has been used previously such as Woods-Saxon potential with spin-orbit and coulomb potentials [1] and M3Y interaction model [2]. Solution of the Schrodinger equation including the above potentials has been done by the numerical methods in the above-mentioned works. This is because analytical solution of these equations is not possible. However, some theoretical groups have tried to solve these Schrodinger equations analytically. For example, Pahlavani et al. have solved the Schrodinger equation including Woods-Saxon potential with spin-orbit and centrifugal terms by Nikiforov-Uvarov method [3]. They did not include the coulomb term to their calculations. Adding the coulomb potential (here for $r<R_{C} ; R_{C}=$ spherical nucleus radius) and solution of the Schrodinger equation by NikiforovUvarov method is the main goal of the present work. In our previous works we have used the NU method to solve the Schrodinger equation with different potentials such as angle-dependent potential [4], Energy-dependent potential [5]; Dirac equation with NU method such as Hartmann potential [6]; Duffin-Kemmer-Petiau (DKP) equation with NU method such as Woods-Saxon potential [7], Hulthen vector potential [8] and Klein-Gordon equation with NU method such as energy-dependent potential [9]. Here, we have extended the Ref. [3] by adding a coulomb term to its potential. Adding this potential enhances the difficulty of the problem very much. Thus, we had to change the transformations and other formulas of the Ref. [3] to be able to solve the Schrodinger equation. Finally, energy eigenvalues and corresponding eigenfunctions obtained. 


\section{Parametric NU method}

This powerful mathematical tool could be used to solve the second-order differential equations. Considering the following differential equation [10-12]

$\psi_{n}^{\prime \prime}(s)+\frac{\tilde{\tau}(s)}{\sigma(s)} \psi_{n}^{\prime}(s)+\frac{\tilde{\sigma}(s)}{\sigma(s)^{2}} \psi_{n}(s)=0$,

where $\sigma(z)$ and $\tilde{\sigma}(z)$ are polynomials of second order at most, and $\tilde{\tau}(z)$ is a first-order polynomial. To make the application of the NU method simpler and the checking of the validity of solution unnecessary, we present a shortcut for the method. We begin the method by writing the general form of the Schrodinger-like Eq. (1) as

$$
\begin{aligned}
\psi_{n}^{\prime \prime}(s) & +\left(\frac{c_{1}-c_{2} s}{s\left(1-c_{3} s\right)}\right) \psi_{n}^{\prime}(s) \\
& +\left(\frac{-p_{2} s^{2}+p_{1} s-p_{0}}{s^{2}\left(1-c_{3} s\right)^{2}}\right) \psi_{n}(s)=0,
\end{aligned}
$$

where the wave functions $\psi_{n}(s)$ satisfies

$\psi_{n}(s)=\phi(s) y_{n}(s)$.

By comparing Eq. (3) with its counterpart Eq. (2), one can obtain

$$
\begin{aligned}
& \tilde{\tau}(s)=c_{1}-c_{2} s, \quad \sigma(s)=s\left(1-c_{3} s\right), \\
& \tilde{\sigma}(s)=-p_{2} s^{2}+p_{1} s-p_{0} .
\end{aligned}
$$

According to the NU method [10], one can obtain the bound state energy equation as [11, 12]

$$
\begin{aligned}
& c_{2} n-(2 n+1) c_{5}+(2 n+1)\left(\sqrt{c_{9}}+c_{3} \sqrt{c_{8}}\right) \\
& \quad+n(n-1) c_{3}+c_{7}+2 c_{3} c_{8}+2 \sqrt{c_{8} c_{9}}=0,
\end{aligned}
$$

where

$$
\begin{aligned}
c_{4} & =\frac{1}{2}\left(1-c_{1}\right), \\
c_{5} & =\frac{1}{2}\left(c_{2}-2 c_{3}\right), \\
c_{6} & =c_{5}^{2}+p_{2}, \\
c_{7} & =2 c_{4} c_{5}-p_{1} \\
c_{8} & =c_{4}^{2}+p_{0}, \\
c_{9} & =c_{3}\left(c_{7}+c_{3} c_{8}\right)+c_{6} .
\end{aligned}
$$

In addition, we also find that:

$$
\begin{aligned}
& \rho(s)=s^{c_{10}}\left(1-c_{3} s\right)^{c_{11}} \\
& \varphi(s)=s^{c_{12}}\left(1-c_{3} s\right)^{c_{13}} \\
& c_{12}>0, c_{13}>0 \\
& y_{n}(s)=P_{n}^{\left(c_{10}, c_{11}\right)}\left(1-2 c_{3} s\right), c_{10}>-1, c_{11}>-1
\end{aligned}
$$

are necessary in calculating the wave functions $\psi_{n l}(s)=N_{n l} s^{c_{12}}\left(1-c_{3} s\right)^{c_{13}} P_{n}^{\left(c_{10}, c_{11}\right)}\left(1-2 c_{3} s\right)$

where $P_{n}^{(\mu, v)}(x), \mu>-1, v>-1, x \in[-1,1]$ are Jacobi polynomials. All undefined constant parameters are as follows [13]:

$$
\begin{aligned}
& c_{10}=c_{1}+2 c_{4}+2 \sqrt{c_{8}} \\
& c_{11}=1-c_{1}-2 c_{4}+\frac{2}{c_{2}} \sqrt{c_{9}} \\
& c_{12}=c_{4}+\sqrt{c_{8}} \\
& c_{13}=-c_{4}+\frac{1}{c_{3}}\left(\sqrt{c_{9}}+c_{5}\right),
\end{aligned}
$$

where

$c_{3} \neq 0, c_{13}>0, c_{12}>0, s \in\left[1,1 / c_{13}\right]$ and $\quad c_{13} \neq 0$.

\section{Solutions of Schrodinger equation}

We treat ${ }^{17} \mathrm{~F}$ as the combination of a proton and an inert ${ }^{16} \mathrm{O}$ core with spin 0 [1]. Schrodinger equation for the $\mathrm{N}$ particles which interact with each other can be written as $[17,18]$ :

$$
\begin{aligned}
& \frac{\hbar^{2}}{2 \mu}\left[\frac{\mathrm{d}^{2}}{\mathrm{~d} r^{2}}+\frac{D-1}{r} \frac{\mathrm{d}}{\mathrm{d} r}-\frac{l(l+D-2)}{r^{2}}\right] R(r)+V(r) R(r) \\
& \quad=\operatorname{ER}(r),
\end{aligned}
$$

where $D=3 N-3$. Here, $D, l$, and $N$ denote, respectively, the space dimension, total angular momentum and the number of particles, $\mu$ is one of particle masses, and $x$ is the hyper-radius. If we write this equation for ${ }^{17} \mathrm{~F}$ as the combination of a proton and an inert ${ }^{16} \mathrm{O}$ core with spin 0 (we have two-particle systems. Thus, $N=2$ and then we find $D=3$ ), we find the radial part of the Schrodinger equation as,

$\frac{\mathrm{d}^{2} R(r)}{\mathrm{d} r^{2}}+\frac{2}{r} \frac{\mathrm{d} R(r)}{\mathrm{d} r}+\frac{2 \mu}{\hbar^{2}}\left[E-V(r)-\frac{\hbar^{2} l(l+1)}{2 \mu r^{2}}\right] R(r)=0$.

The potential profile contain the following terms.

1. Woods-Saxon:

$V_{\mathrm{WS}}=\frac{-V_{0}}{1+e^{\frac{\left(r-R_{0}\right)}{a}}}$

We have used $V_{0}=42.71 \mathrm{MeV}$ for ${ }^{17} \mathrm{~F}$ atom [3]. There, $V_{0}$ is defined as $40.5+0.13 A \mathrm{MeV}$, where $A$ is the mass number of nuclei (here 17 for ${ }^{17} \mathrm{~F}$ ). $R_{0}$ is radius of the spherical nucleus and $a=0.65 \mathrm{fm}$ is surface diffuseness.

Woods-Saxon potential has vast applications in description of both spherical and deformed nuclei in nuclear and particle physics. Analytic solution of Schrödinger 
equation with Woods-Saxon potential is very useful which provide for us invaluable theoretical results. DKP equation is also solved analytically by means of NU method a vector deformed Woods-Saxon potential [18]. However, scientist have solved the Woods-Saxon potential for $S$ states $(l=0)$, exactly and also by some approximation they have also found the solutions for any $l$ states. [19-22]

2. Spin-orbit term as,

$V_{L S}(r)=V_{L S}^{(0)}\left(\frac{r_{0}}{\hbar}\right)^{2} \frac{1}{r}\left[\frac{\mathrm{d}}{\mathrm{d} r} \frac{1}{1+e^{\frac{\left(r-R_{0}\right)}{a}}}\right](\vec{L} \cdot \vec{S})$

where we have used $V_{L S}^{(0)}=0.44 V_{0}$.

3. Coulomb term,

$V_{C}(r)= \begin{cases}\frac{e^{2}}{R_{C}}\left[3-\left(\frac{r}{R_{C}}\right)^{2}\right] & r \leq R_{C} \\ \frac{8 e^{2}}{r} & r \leq R_{C}\end{cases}$

Here we have solved the Schrodinger equation for $r<R_{C}$. By using of the change of variable as $\psi(r)=r R(r)$ we have,

$\frac{\mathrm{d} \psi}{\mathrm{d} r}=R(r)+r \frac{\mathrm{d} R(r)}{\mathrm{d} r}$.

Then the Schrodinger equation becomes,

$\frac{\mathrm{d}^{2} \psi(r)}{\mathrm{d} r^{2}}+\frac{2 \mu E}{\hbar^{2}} \psi(r)-\frac{2 \mu V(r)}{\hbar^{2}} \psi(r)-\frac{l(l+1)}{r^{2}} \psi(r)=0$.

Now, complete Schrodinger equation reads,

$$
\begin{aligned}
& \frac{\mathrm{d}^{2} \psi(r)}{\mathrm{d} r^{2}}+\frac{2 \mu E}{\hbar^{2}} \psi(r) \\
& -\frac{2 \mu}{\hbar^{2}}\left[\frac{-V_{0}}{1+e^{\frac{\left(r-R_{0}\right)}{a}}}+V_{L S}^{(0)} r_{0}^{2} \frac{1}{r}\left(\frac{\mathrm{d}}{\mathrm{d} r} \frac{1}{1+e^{\frac{\left(r-R_{0}\right)}{a}}}\right)\right. \\
& \left.\quad \times\left(j(j+1)-l(l+1)-\frac{3}{4}\right)+\frac{3 e^{2}}{\pi \varepsilon_{0} R_{C}}-\frac{e^{2} r^{2}}{\pi \varepsilon_{0} R_{C}^{3}}\right] \psi(r) \\
& \quad-\frac{l(l+1)}{r^{2}} \psi(r)=0 .
\end{aligned}
$$

We need another change of variable as $s=e^{-\delta r}$, which leads,

$\frac{\mathrm{d} \psi(r)}{\mathrm{d} r}=\frac{\mathrm{d} \psi(r)}{\mathrm{d} s} \times \frac{\mathrm{d} s}{\mathrm{~d} r}=-\delta s \frac{\mathrm{d} \psi(r)}{\mathrm{d} s}$

$\frac{\mathrm{d}^{2} \psi(r)}{\mathrm{d} r^{2}}=\delta^{2} s^{2} \frac{\mathrm{d}^{2} R(s)}{\mathrm{d} s^{2}}+\delta^{2} s \frac{\mathrm{d} R(s)}{\mathrm{d} s}$.

We define $\delta=\frac{1}{a}, q=e^{\delta R_{0}}, V_{0}^{\prime}=V_{0} q$ and $V_{L S}^{\prime 0}=V_{L S}^{0} q$. We have used the following approximation too,

$\frac{1}{r^{2}} \approx \delta^{2}\left(\frac{e^{-\delta r}}{1-e^{-\delta r}}\right)^{2}$
To find the best value of the $\delta$ we have changed the $\delta$ and plotted both side of the above equation several times and found the best $\delta$ to be $0.4 \mathrm{fm}^{-1}$. Figure 1 shows two sides of the above equation to obtain $\delta$. By using the later change of variable the Schrodinger equation reads,

$$
\begin{aligned}
& \delta^{2} s^{2} \frac{\mathrm{d}^{2} R(s)}{\mathrm{d} s^{2}}+\delta^{2} s \frac{\mathrm{d} R(s)}{\mathrm{d} s}+\left(\frac{2 \mu E}{\hbar^{2}}+\frac{2 \mu V_{0}^{\prime}}{\hbar^{2}}\left(\frac{s}{1+q s}\right)\right. \\
& +\frac{\mu r_{0}^{2} V_{L S}^{\prime 0} \delta^{2}}{\hbar^{2}}\left(j(j+1)-l(l+1)-\frac{3}{4}\right) \frac{s^{2}}{(1-s)(1+q s)^{2}} \\
& \left.-\frac{6 \mu e^{2}}{\pi \varepsilon_{0} \hbar^{2} R_{C}}+\frac{2 \mu e^{2}}{\pi \varepsilon_{0} \hbar^{2} R_{C}^{3} \delta^{2}} \frac{(1-s)^{2}}{s^{2}}-l(l+1) \delta^{2} \frac{s^{2}}{(1-s)^{2}}\right) \\
& \times R(s)=0 .
\end{aligned}
$$

After some simplifications we have,

$$
\begin{aligned}
& \frac{\mathrm{d}^{2} R(s)}{\mathrm{d} s^{2}}+\frac{1}{s} \frac{\mathrm{d} R(s)}{\mathrm{d} s}+\left(\frac{\frac{2 \mu E}{\hbar^{2} \delta^{2}}(1-s)^{2}+\frac{2 \mu V_{0}^{\prime}}{\hbar^{2} \delta^{2}} \frac{s(1-s)^{2}}{1+q s}}{s^{2}(1-s)^{2}}\right. \\
& +\frac{\frac{\mu r_{0}^{2} V_{L S}^{\prime 0}}{\hbar^{2}}\left(j(j+1)-l(l+1)-\frac{3}{4}\right) \frac{s^{2}(1-s)}{(1+q s)^{2}}-\frac{6 \mu e^{2}}{\pi \varepsilon_{0} \hbar^{2} R_{C} \delta^{2}}(1-s)^{2}}{s^{2}(1-s)^{2}} \\
& \left.+\frac{\frac{2 \mu e^{2}}{\pi \varepsilon_{0} \hbar^{2} R_{C}^{3} \delta^{4}} \frac{(1-s)^{4}}{s^{2}}-l(l+1) s^{2}}{s^{2}(1-s)^{2}}\right) R(s)=0 .
\end{aligned}
$$

Here, we present a new procedure with which we can convert the Schrodinger equation with any shape of the potential profile into the NU type equation. To use the NU method we convert the numerator of the potential term to a second-order polynomial. For this purpose we have used the following approximations which we have found through numerical fitting,

$$
\begin{aligned}
\frac{s(1-s)^{2}}{1+q s}= & 0.006595579 s^{2}-0.0132710857 s \\
& +0.0067413175 .
\end{aligned}
$$

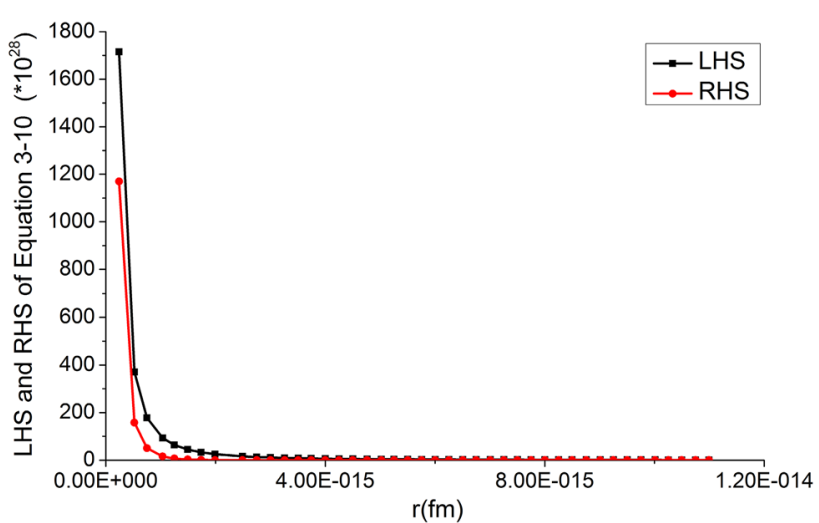

Fig. 1 Left hand side (LHS) and right hand side (RHS) of the Eq. (23) to obtain $\delta$ 
Figure 2a shows the two sides of the equation above. As it is clear, this approximation is extremely good. We have also used from

$$
\begin{aligned}
\frac{s^{2}(1-s)}{(1+q s)^{2}}= & -3.3579988 \times 10^{-9} s^{2}-0.432019240084 \\
& \times 10^{-4} s+0.4341808999 \times 10^{-4} .
\end{aligned}
$$

Figure $2 b$ shows the two sides of the equation above. As it is clear, this approximation is extremely good. We have also used from

$$
\frac{(1-s)^{4}}{s^{2}}=0.981 s^{2}-3.537 s+3.4 .
$$

Figure $2 \mathrm{c}$ shows the two sides of the equation above. As it is clear, this approximation is extremely good. To convert the last Schrodinger equation to the N-U type Schrodinger equation,

$\psi_{n}^{\prime \prime}(s)+\frac{\tilde{\tau}(s)}{\sigma(s)} \psi_{n}^{\prime}(s)+\frac{\tilde{\sigma}(s)}{\sigma(s)^{2}} \psi_{n}(s)=0$

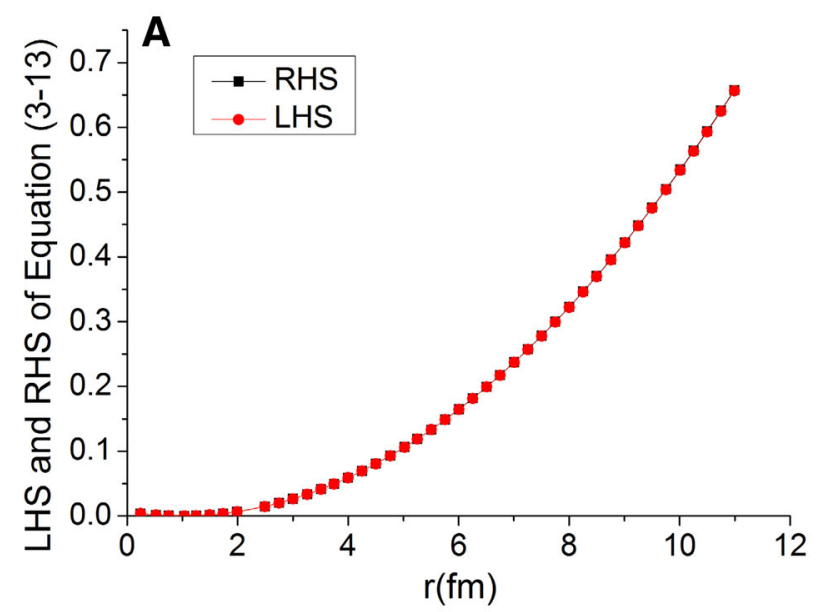

$\psi_{n}^{\prime \prime}(s)+\left(\frac{1-s}{s(1-s)}\right) \psi_{n}^{\prime}(s)+\left(\frac{-\gamma s^{2}+\beta s-\varepsilon^{2}}{s^{2}(1-s)^{2}}\right) \psi_{n}(s)=0$

We define three parameters $\varepsilon, \beta, \gamma$ as follows:

$$
\begin{aligned}
-\gamma= & \frac{2 \mu E}{\hbar^{2} \delta^{2}}+\frac{2 \mu V_{0}^{\prime}}{\hbar^{2} \delta^{2}}(0.006595579) \\
& +\frac{\mu V_{L S}^{\prime 0} r_{0}^{2}\left(j(j+1)-l(l+1)-\frac{3}{4}\right)}{\hbar^{2}} \\
& \times\left(-3.3579988 \times 10^{-9}\right) \\
& -\frac{6 \mu e^{2}}{\pi \varepsilon_{0} \hbar^{2} R_{C} \delta^{2}}+\frac{2 \mu e^{2}}{\pi \varepsilon_{0} \hbar^{2} R_{C}^{3} \delta^{4}}(0.981)-l(l+1)
\end{aligned}
$$

$$
\begin{aligned}
\beta= & \frac{4 \mu E}{\hbar^{2} \delta^{2}}+\frac{2 \mu V_{0}^{\prime}}{\hbar^{2} \delta^{2}}(0.0132710857) \\
& +\frac{\mu V_{L S}^{\prime 0} r_{0}^{2}\left(j(j+1)-l(l+1)-\frac{3}{4}\right)}{\hbar^{2}} \\
& \times\left(0.432019240084 \times 10^{-4}\right) \\
& +\frac{12 \mu e^{2}}{\pi \varepsilon_{0} \hbar^{2} R_{C} \delta^{2}}-\frac{2 \mu e^{2}}{\pi \varepsilon_{0} \hbar^{2} R_{C}^{3} \delta^{4}}(3.537)
\end{aligned}
$$

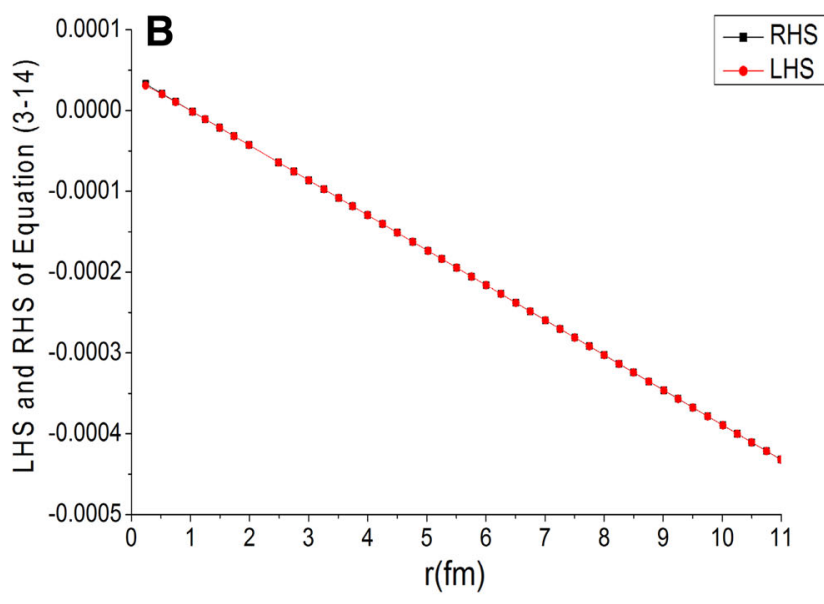

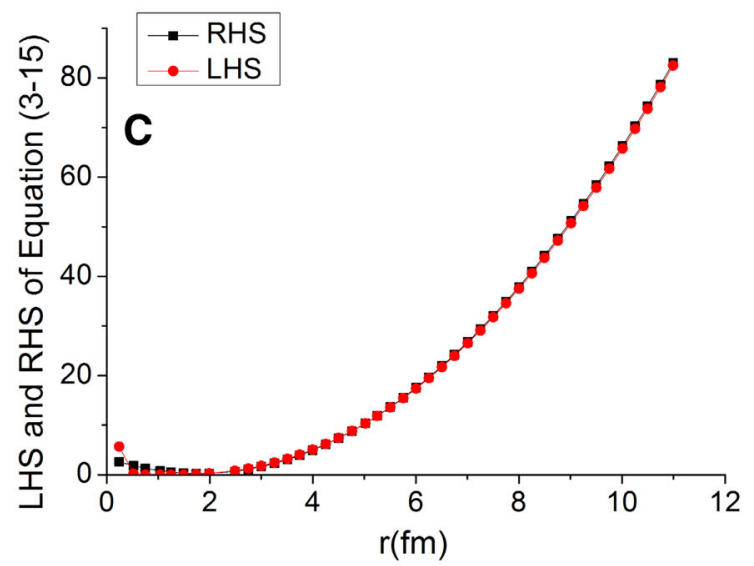

Fig. 2 a Left hand side (LHS, filled circle line) and right hand side (RHS, filled square line) of the Eq. (26) to show the accuracy of the approximation (27). b Same as a but for Eq. (27). b Same a but for Eq. (28) 


$$
\begin{aligned}
-\varepsilon^{2}= & \frac{2 \mu E}{\hbar^{2} \delta^{2}}+\frac{2 \mu V_{0}^{\prime}}{\hbar^{2} \delta^{2}}(0.0067413175) \\
& +\frac{\mu V_{L S}^{\prime 0} r_{0}^{2}\left(j(j+1)-l(l+1)-\frac{3}{4}\right)}{\hbar^{2}} \\
& \times\left(0.4341808999 \times 10^{-4}\right) \\
& -\frac{6 \mu e^{2}}{\pi \varepsilon_{0} \hbar^{2} R_{C} \delta^{2}}+\frac{6.8 \mu e^{2}}{\pi \varepsilon_{0} \hbar^{2} R_{C}^{3} \delta^{4}}
\end{aligned}
$$

Now we write $\beta, \gamma$ as function of $\varepsilon$,

$$
\begin{aligned}
\gamma= & \varepsilon^{2}+\frac{\mu V_{0}^{\prime}}{\hbar^{2} \delta^{2}}(0.000291477) \\
& +\frac{\mu V_{L S}^{\prime 0} r_{0}^{2}\left(j(j+1)-l(l+1)-\frac{3}{4}\right)}{\hbar^{2}} \\
& \left(0.434214479888 \times 10^{-4}\right) \\
& +\frac{4.838 \mu e^{2}}{\pi \varepsilon_{0} \hbar^{2} R_{C}^{3} \delta^{4}}+l(l+1) \rightarrow \gamma=\varepsilon^{2}+A \\
\beta= & 2 \varepsilon^{2}+\frac{0.0004230986 \mu V_{0}^{\prime}}{\hbar^{2} \delta^{2}} \\
& +\frac{\mu V_{L S}^{\prime 0} r_{0}^{2}\left(j(j+1)-l(l+1)-\frac{3}{4}\right)}{\hbar^{2}} \\
& \times\left(0.436342559716 \times 10^{-4}\right) \\
& +\frac{6.526 \mu e^{2}}{\pi \varepsilon_{0} \hbar^{2} R_{C}^{3} \delta^{4}} \rightarrow \beta=2 \varepsilon^{2}+B
\end{aligned}
$$

Based on what we have described above in the N-U section, we can find the following second-order polynomial:

$$
\begin{gathered}
n-(2 n+1)\left(-\frac{1}{2}\right)+(2 n+1)\left(\sqrt{\gamma-\beta+\varepsilon^{2}+\frac{1}{4}}+\varepsilon\right) \\
+n(n+1)-\beta+2 \varepsilon^{2}+2 \varepsilon \sqrt{\gamma-\beta+\varepsilon^{2}+\frac{1}{4}}=0 .
\end{gathered}
$$

After some simplifications we have,

$$
\begin{aligned}
& {\left[4\left(A-B+\frac{1}{4}\right)-(2 n+1)^{2}\right] \varepsilon^{2}+2(2 n+1)\left[2 A-B-n^{2}-n\right] \varepsilon} \\
& +\left[(2 n+1)^{2}\left(A-B+\frac{1}{4}\right)-\left(n^{2}+n+\frac{1}{2}-B\right)^{2}\right]=0 \\
& h_{2} \varepsilon^{2}+h_{1} \varepsilon+h_{0}=0 .
\end{aligned}
$$

By solving this equation and obtaining the $\varepsilon$ we will wined the energies $E$ as,

$$
\begin{aligned}
E_{n l j}= & \frac{-\hbar^{2} \delta^{2}}{2 \mu}\left(\varepsilon^{2}+\frac{2 \mu V_{0}^{\prime}}{\hbar^{2} \delta^{2}}(0.0067413175)\right. \\
& +\frac{\mu V_{L S}^{\prime 0} r_{0}^{2}\left(j(j+1)-l(l+1)-\frac{3}{4}\right)}{\hbar^{2}}\left(0.4341808999 \times 10^{-4}\right) \\
& \left.-\frac{6 \mu e^{2}}{\pi \varepsilon_{0} \hbar^{2} R_{C} \delta^{2}}+\frac{6.8 \mu e^{2}}{\pi \varepsilon_{0} \hbar^{2} R_{C}^{3} \delta^{4}}\right)
\end{aligned}
$$

Table 1 Comparison of the energies obtained in this work with ab initio computation [14] and experimental values [16]

\begin{tabular}{llll}
\hline${ }^{17} F$ & & \\
\hline$E_{S O}$ & $\frac{1}{2}^{+}$ & $\frac{5}{2}^{+}$ & \\
\hline 5.000 & -0.105 & -0.600 & Exp. [16] \\
3.7 & -0.082 & 0.11 & GHF [14] \\
& 0.023 & 0.71 & GHF [14] absolute error \\
- & -0.1968 & -0.937 & Our work \\
& 0.0918 & 0.337 & Our absolute error \\
\hline
\end{tabular}

All energies stated in the $\mathrm{MeV}$

Ground state energy of the ${ }^{17} \mathrm{~F}$ can be obtained by using of $j=\frac{5}{2} n=0, l=2$ and the first excited states can be calculated by using of the $j=\frac{1}{2} n=1, l=0$. Table 1 shows the results obtained in this work and Refs. [14, 16]. As it is clear from the Table 1, good accuracy in our work has been achieved. However, we have calculated the $\frac{1}{2}^{+}$state energy with more accuracy (our absolute error $=0.023 \mathrm{MeV}$, Hagen et al. absolute error $=0.0918 \mathrm{MeV}$ ) while Hagen et al. have calculated the $\frac{5}{2}^{+}$state energy with higher accuracy (our absolute error $=0.71 \mathrm{MeV}$, Hagen et al. absolute error $=0.0337 \mathrm{MeV}$ ). Now, the wave functions can be obtained through,

$\psi(s)=y(s) \phi(s)$

$\rho(s)=s^{1+2 \varepsilon}(1-s)^{2 \sqrt{\gamma-\beta+\varepsilon^{2}+\frac{1}{4}}}$

$\phi(s)=s^{\varepsilon}(1-s)\left(\sqrt{\gamma-\beta+\varepsilon^{2}+\frac{1}{4}-\frac{1}{2}}\right)$

$y_{n}(s)=P_{n}^{\left(1+2 \varepsilon, 2 \sqrt{\gamma-\beta+\varepsilon^{2}+\frac{1}{4}}\right)}(1-2 s)$

$\psi_{n l}(s)=N_{n l} s^{\varepsilon}(1-s)^{\sqrt{\gamma-\beta+\varepsilon^{2}+\frac{1}{4}}+\frac{1}{2}} \times P_{n}^{\left(1+2 \varepsilon, 2 \sqrt{\gamma-\beta+\varepsilon^{2}+\frac{1}{4}}\right)}(1-2 s)$,

where $P_{n}^{(\mu, v)}(x), \mu>-1, v>-1, x \in[-1,1]$ are Jacobi polynomials. $5 / 2^{+}$state and $1 / 2^{+}$state wave functions are presented in the Fig. 3a, b, respectively. As we can see in these figures, good agreement exists. We have used the parameters of the [3], since they lead to the energies closer to the experimental values which we have presented in the Table 1.

\section{Conclusion}

In this study, the non-relativistic radial Schrodinger equation solved for Wood-Saxon potential together with coulomb potential (for $r<R_{C} ; R_{C}=$ spherical nucleus radius), spin-orbit interaction and centrifugal term through a new hybrid numerical fitting Nikiforov-Uvarov method. For this 


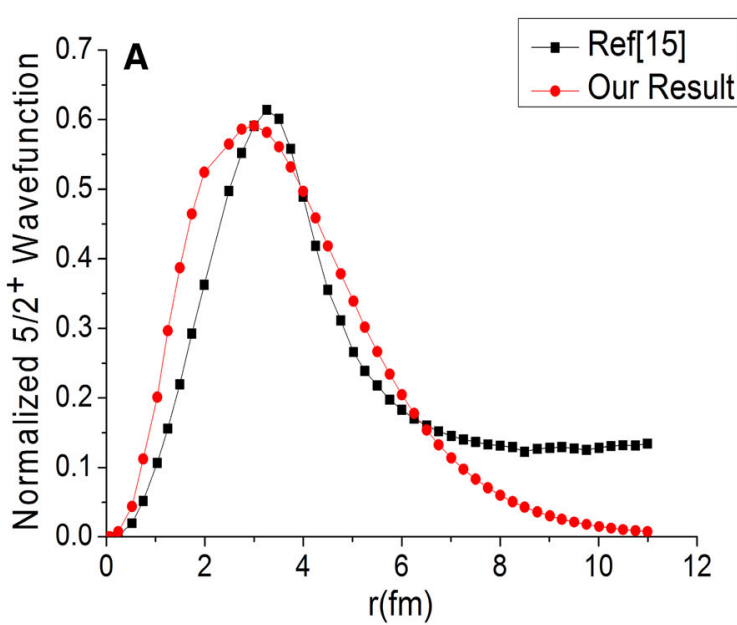

Fig. 3 a Normalized $5 / 2^{+}$state wave function which we have calculated (filled circle line) compared with [15] (filled square line) as a function of the radius $r(\mathrm{fm})$. b Normalized $1 / 2^{+}$state wave function

purpose, by using approximate expansion of $1 / r^{2}$ different than that used by Pahlavani et al., Schrodinger equation has been transformed to the analytically solvable differential equation of NU method. Good agreement with experimental values is obtained. We have calculated the $1 / 2^{+}$state energy with more accuracy (our relative error $=0.023 \mathrm{MeV}$ and Hagen et al. relative error $=0.0918 \mathrm{MeV}$ ), while Hagen et al. have calculated the $5 / 2^{+}$state energy with higher accuracy (our relative error $=0.71 \mathrm{MeV}$ and Hagen et al. relative error $=0.0337 \mathrm{MeV}$ ). Our obtained wave functions are in agreement with Kim et al.'s too.

Open Access This article is distributed under the terms of the Creative Commons Attribution 4.0 International License (http://creative commons.org/licenses/by/4.0/), which permits unrestricted use, distribution, and reproduction in any medium, provided you give appropriate credit to the original author(s) and the source, provide a link to the Creative Commons license, and indicate if changes were made.

\section{References}

1. Esbensen, H., Bertsch, G.F.: Higher-order effects in the two-body breakup of 17F. Nucl. Phys. A 706, 383 (2002)

2. Bertulani, C.A.: RADCAP: A potential model tool for direct capture reactions. Comp. Phys. Commun. 156, 123 (2003)

3. Pahlavani, M.R., Alavi, S.A.: Solutions of woods-saxon potential with spin-orbit and centrifugal terms through Nikiforov-Uvarov method. Commun. Theor. Phys. 58, 739 (2012)

4. Rajabi, A.A., Hamzavi, M.: A new Coulomb ring-shaped potential via generalized parametric Nikiforov-Uvarov method. J. Theor. Appl. Phys. 7, 17 (2013)

5. Hassanabadi, H., Zarrinkamar, S., Rajabi, A.A.: Exact solutions of D-dimensional Schrödinger equation for an energy-dependent potential by NU method. Commun. Theor. Phys. 55, 541 (2011)

6. Hamzavi, M., Hassanabadi, H., Rajabi, A.A.: Exact solutions of Dirac equation with Hartmann potential by Nikoforov-Uvarov method. Int. J. Mod. Phys. E 19, 2189 (2010)

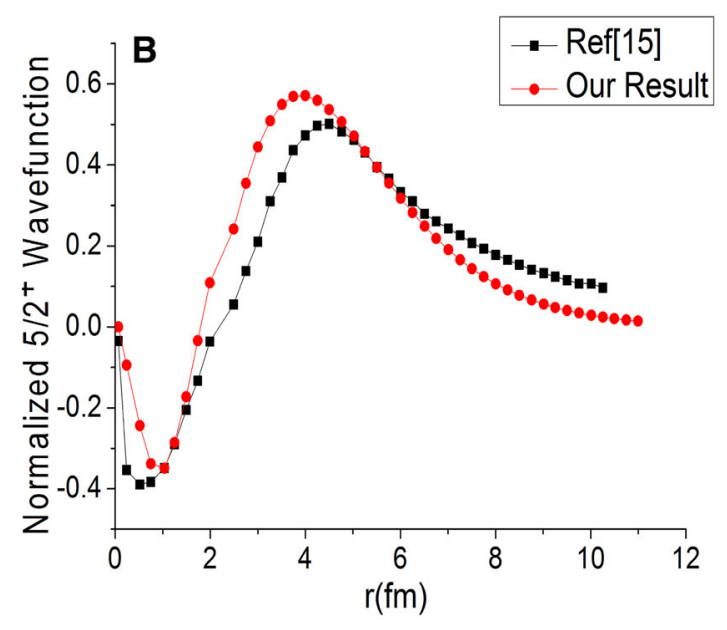

which we have calculated (filled circle line) compared with Ref. [15] (filled square line) as a function of the radius $r(\mathrm{fm})$

7. Oudi, R., Hassanabadi, S., Rajabi, A.A., Hasanabadi, H.: Approximate bound state solutions of DKP equation for any $\mathbf{J}$ state in the presence of woods-saxon potential. Commun. Theor. Phys. 57, 15 (2012)

8. Zarrinkamar, S., Rajabi, A.A., Yazarloo, B.H., Hassanabadi, H.: An approximate solution of the DKP equation under the Hulthén vector potential. Chin. Phys. C 37, 023101 (2013)

9. Hassanabadi, H., Zarrinkamar, S., Hamzavi, H., Rajabi, A.A.: Exact solutions of D-dimensional Klein-Gordon equation with an energy-dependent potential by using of Nikiforov-Uvarov method. Arab. J. Sci. Eng. 37, 209 (2012)

10. Nikiforov, A.F., Uvarov, V.B.: Special functions of mathematical physics. Birkhausr, Berlin (1988)

11. Ikhdair, S.M.: Bound states of the Klein-Gordon equation for vector and scalar general Hulthen-type potentials in D-dimension. Int. J. Mod. Phys. C 20, 25 (2009)

12. Tezcan, C., Sever, R.: A general approach for the exact solution of the Schrödinger equation. Int. J. Theor. Phys. 48, 337 (2009)

13. Wang, Z.B., Zhang, M.C.: Spinless particles in the field of unequal scalar-vector Yukawa potentials. Acta Phys. Sin. 56, 3688 (2007). (in Chinese)

14. Hagen, G., Papenbrock, T., Hjorth-Jensen, M.: Ab Initio Computation of the 17F Proton Halo State and Resonances in $\mathrm{A}=17$ Nuclei. Phys. Rev. Lett. 104, 182501 (2010)

15. Kim, K.-H.: Astrophysical S factors of the $16 \mathrm{O}(\mathrm{p}, \gamma) 17 \mathrm{~F}$ reaction at energies applicable in stellar cores. J. Korean Phys. Soc. 43, 691 (2003)

16. Tilley, D.R., Weller, H.R., Cheves, C.M.: Energy levels of light nuclei A = 16-17. Nucl. Phys. A 564, 1 (1993)

17. Rajabi, A.A.: Exact analytical solution of the Schrödinger equation for an N-identical body-force system. Few Body Syst. 37, 197 (2005)

18. Hamzavi, M., Ikhdair, S.M.: Any J-state solution of the DKP equation for a vector deformed Woods-Saxon potential. FewBody Syst. (2012). arXiv:1205.0938v2

19. Feizi, H., Rajabi, A.A., Shojaei, M.R.: Supersymmetric solution of the Schrödinger equation for Woods-Saxon potential by using the Pekeris approximation. Acta. Phys. Pol. B 42, 2143 (2011)

20. Ikhdair, S.M., Sever, R.: Exact polynomial solution of non-symmetric and non-Hermitian modified Woods-Saxon potential by the Nikiforov-Uvarov method. Int. J. Theor. Phys. 46, 1643 (2007) 
21. Berkdemir, C., Berkdemir, A., Sever, R.: Polynomial solutions of the Schrödinger equation for the generalized Woods-Saxon potential. Phys. Rev. C 72, 027001 (2005)
22. Berkdemir, C., Berkdemir, A., Sever, R.: Polynomial solutions of the Schrödinger equation for the generalized Woods-Saxon potential. Phys. Rev. C 74, 039902 (2006) 